\title{
Author Index, Vol. 19
}

Armstrong, D. 260 Aronyk, K, E. 101

Barlett, S.P. 63 Barnes, P.D. 25 Basauri, Ll 109 Bauer, S. 256 Bazmi, B. 243 Becker, L.E. 186 Bruce, D.A. 170, 171 Buncic, J.R. 260

Camel, P.W. 276 Canady, A.I. 202 Carlson, C.V. 233 Chadduck, W.M. 81 Chapman, P.H. 267 Cheek,|W.R. 89 Cohen,|A.R. 127 Cohen, B.H. 171 ContiReed, U. 180

D’Angio, G. 171 Das, S.K. 73 Dauser, R.C, 243 Davis, K.R. 267 Diament, AJ. 180 Douglass, E.C. 113 Drake, J.M. 151, 186,260 Duhaime, A.-C. 63

Ecklund, J. 196 Epstein, F.J. 300 Ersahin, Y. 31 Eviatar, L. 84

Ferreira, J. 84 Fessler, R.D. 202

Gajjar, A. 113 George, R.E. 89

Gherpelli, J.L.D. 180 Goodman, JJ.C. 89 Greenberg, M. 186 Greif, L.K. 276 Grossman, R. 84 Gupta,|R.K. 206

Hahn, Y.S. 135,225 Haines, D.E. 73 Heideman, R.L. 113 Hendren, W.H. Ill 25 Hendricks, E.B. 151 Herman, J.M. 243 Higgins, M.J. 40 Hoffman, HJ. 151,186,303 Horowitz, M.E. 113 Humphreys, R.P. 119,151, $169,170,186$

Jay, V. 93 Jenkin, D. 186 Jenkins, J.J. 113 Johnson, D. 111

Kalia, K, K. 78 Kamio, M. 121 Klein, D. Ill Klein, M.D. 202 Kovnar, E.H. 113 Kun, L.E. 113

Langston, J.A. 113 Laurent,,J.P. 89 Lizana, C, 109 Luerssen, T.G. 170,215 Lussenhop, A. 111

Macaulay, R.J.B. 93 Machado de Almeida, G.G. 180 
McLone, D.G. 6,101, 135,

233, 243 Madsen, J.R. 256 Mahapatra, A.K. 34 Mallette, R.A. 73 Mandabach, M. 225 Markakis, D. 63 Marmarou, A. 216 Marquardt, M.D. 250 Matsumura, H. 21 Matushita, H. 180 Menezes, A.H. 165,224 Mickle, J.P. 143 Montgomery, C.T. 209 Morii, S. 21 Murovic, J.A. 151 Mutluer, S. 31

Nakajima, M. 121 Nakamura, N. 121 Numoto, T. 121

Packer,|R.J. 119, 171 Pang, D. 78 Parent,|A.D. 73 Piatt, J.H., Jr. 225 Posnick, J.C| 151, 160

Quisling, R.G. 143 Quisling, S.G. 143

Reigel, D.H. 169,243 Rekate, H.L. 15 Roloson, G.J. 81 Rorke, L.B. 57, 171, 196 Rosemberg, S. 180 Ruge, J.R. 225 Rushing, E.J. 57 Rutka, J.T. 186 Ryken, T.C! 165 
Sainte-Rose, C. 156 Sanford, R.A. 113 Sathi, S. 256 Schneider, S. 84 Schut, L. 171, 196 Scott, R.M. 25, 256 Selman, J.M. 109 Shapiro,IK 216,223 Sharma, K. 206 Shi, S.-R. 250 Shiminski-Maher, T. 104 Shulman, K. 216 Siegel, K, R. 171 Simpson, R.K, Jr. 89 Sladky, J.T. 93 Storrs, B.B. 215,243 Sutton, L.N. 57,63, 171 Swift,|D.M. 78

Tanaka, H. 121 Tanaka, J. 121 Tandon, D.A. 34 Tomita, T. 6 Tredwell, S.J. 224 Tsubura, A. 21

Venes, J. 1 Ventureyra, E.C.G. 40

Warf, B.C. 25 Wells, M.D. 260 Winfield, J.A. 209 Wisoff, J.H. 215

Yamanouchi, Y. 21 Yasuda, T. 21 Yasue, M. 121 